\title{
Corrigendum: CRT1 is a nuclear-translocated MORC endonuclease that participates in multiple levels of plant immunity
}

Hong-Gu Kang, Hyong Woo Choi, Sabrina von Einem, Patricia Manosalva, Katrin Ehlers, Po-Pu Liu, Stefanie V. Buxa, Magali Moreau, Hyung-Gon Mang, Pradeep Kachroo, Karl-Heinz Kogel \& Daniel F. Klessig

Nature Communications 3:1297 doi:10.1038/ncomms2279 (2012); Published 18 Dec 2012; Updated 26 Feb 2013

The original version of this Article contained a typographical error in the spelling of the author Hyung-Gon Mang, which was incorrectly given as Hyong-Gon Mang. This has now been corrected in both the PDF and HTML versions of the Article. 